\title{
Tuberculosis: A Review of Current Trends
}

\author{
Sanyaolu $\mathbf{A}^{1 *}$, Schwartz J2, Roberts $\mathbf{K}^{2}$, Evora J2, Dhother $\mathbf{K}^{2}$, \\ Scurto F2, Lamech $\mathrm{S}^{2}$, Rungteranoont $\mathrm{T}^{2}$, Desai V2, Dicks $\mathrm{C}^{2}$, \\ Dimarco $\mathrm{C}^{2}$, Patel S ${ }^{2}$ \\ ${ }^{1}$ Federal Ministry of Health Abuja, Nigeria \\ ${ }^{2}$ Saint James School of Medicine, Anguilla BWI
}

Review Article

Volume 3 Issue 2

Received Date: March 22, 2019

Published Date: May 06, 2019

DOI: $10.23880 /$ eij-16000123

*Corresponding author: Dr. Adekunle Sanyaolu, Federal Ministry of Health, Abuja, Nigeria, Tel: +18134036073;

Email: sanyakunle@gmail.com

\section{Abstract}

Tuberculosis (TB), an old disease caused by the bacteria Mycobacterium tuberculosis is still responsible for more deaths worldwide each year than any other infectious disease, including human immunodeficiency virus (HIV) in spite of availability of effective treatment that has existed for over 50 years since the 1940s. The development of resistance to antibiotics is only one of the many challenges facing the fight to stem this deadly epidemic. Practical factors including a lack of community awareness, access to diagnostic tools, healthcare facilities, and patient supervision and follow-up are compounded by the disease's wide geographic range. A failure of communication and coordination between local and international control units is equally problematic. As we continue to make great strides in medical technology and research, there is the need for a greater effort to mobilize resources and make lasting, focused investments of funding and professional training in areas of endemic TB in order to finally see an end to this bacteria's lasting reign of devastation. This review article looks at the current global trends of the disease from a broader perspective.

Keywords: Tuberculosis (TB); Multiple-drug resistant (MDR-TB); Extensively-drug resistant (XDR-TB); Totallydrug resistant (TDR-TB); Tuberculin purified protein derivative (PPD)

\section{Introduction}

Tuberculosis (TB) has consistently shown a much higher annual mortality rate than HIV or any other infection. This is due to an array of events that begins with the virulence of Mycobacterium tuberculosis, the highly contagious and persistent bacterium responsible for TB infection. Another contributing factor is the ability of these bacteria to develop genetic mutations that confer resistance to a number of formerly effective antibiotics. The World Health Organization (WHO) estimated about 480,000 cases of multiple-drug resistant (MDR-) TB detected worldwide in 2013. MDR$\mathrm{TB}$ and its more resistant sibling, extensively-drug resistant (XDR-) TB, have become increasingly common since successful antibiotic treatments of TB were discovered. Ominously, some experts predict that MDRTB will replace non-resistant TB as the most common form of the disease in the next 50 years [1].

However, there has been a great success in developing effective techniques for preventative care, treatment, and infection control. The annual mortality rate of TB has dropped almost 50\% since 1990, and global health organizations are poised to build upon this positive momentum. It has even been stated that after a decade of public health programs and research, "2015 is a watershed moment in the battle against tuberculosis" 
[2]. Proven care and management methods combined with promising new techniques for better detection and treatment of TB will enable healthcare professionals to continue to make enormous strides in this high-stakes battle. Despite this success, funding for TB control falls far short of that for other infectious diseases. It is clearly evident that present funding and communication gaps are stagnating prevention and control efforts; however targeted investments will pay off in huge dividends in the global campaign to eradicate this debilitating disease [2].

\section{Etiology agent}

\section{Mycobacterium tuberculosis}

M. tuberculosis infection has been known throughout human history. The bacterium is believed to have originated from East Africa. As early humans moved out of East Africa, settling in Europe and Asia, TB infection moved with them and continued to wreak devastation for centuries throughout the known world [3]. Evidence of tubercular decay was seen on the spines of mummies from the Egyptian pre-dynastic era and the Peruvian pre-Columbian era, around 2400 B.C. [4]. Ancient Greeks termed the illness "phthisis." Later, the "Great White Plague" of TB infection raged across Europe for over a century. Throughout this time, the disease was considered almost inevitably fatal, and no effective treatment or cure existed [3].

A milestone occurred when Hermann Heinrich Robert Koch discovered and expounded the etiology of tuberculosis in his presentation "Die Aetiologie der Tuberculose" to the Berlin physiological society. He presented his discoveries on March 24th, 1882, and later received the Nobel Prize in 1905. This was the start of an era of unprecedented advances in the treatment and prevention of this deadly disease [5]. In 1943 another milestone was marked: the first known effective cure for the infection, antibiotic called streptomycin, was discovered in a laboratory at Rutgers University in New Jersey. The first large-scale clinical trial of streptomycin took place at the British Medical Research Council in 1948 and was the first published drug trial to randomize participants. This study set the methodological standard for modern-day randomized, controlled trials. It also was the first time patients showed resistance to streptomycin. Also in 1948, two new anti-tuberculosis agents, thiacetazone and paraaminosalicylic acid, entered the market. When either of these agents was administered with streptomycin, cure rates dramatically increased and acquired resistance in the bacteria decreased $[6,7]$.

Isoniazid was successfully tested and added to the TB regimen in 1951. This was followed by the development of a plethora of new drugs: pyrazinamide and cycloserine in 1952, ethionamide in 1956, rifampin in 1957, and ethambutol in 1962. Rifampin was highly effective and easy to administer, and marked a significant advance in the fight against TB $[7,8]$.

Unfortunately, the widespread use of the new drugs led to the development of bacterial mutations conferring resistance. Resistance to rifampin was observed in $M$. tuberculosis shortly after its initiation as a TB treatment standard. Bacterial resistance to isoniazid also quickly developed, but when other drugs like streptomycin or para-amino salicylic acid were discovered, the resistance was successfully suppressed. These observations led to the regular use of multidrug treatment regimens, an approach that is still considered the standard in treatment of tuberculosis as well as in other diseases [7].

After a series of international clinical trials led by the British Medical Research Council, a four-drug regimen was recommended for use in newly diagnosed tuberculosis patients. The pillars of this regimen were isoniazid and rifampin, the most effective and welltolerated oral agents available, given for a period of 6 to 8 months. This short-course chemotherapy remains the first-line treatment regimen of non-resistant TB today [6].

\section{Pathogenesis}

Mycobacterium tuberculosis is an airborne pathogen. Once inhaled, droplets bearing the mycobacteria settle throughout the airways. Most of the bacilli are trapped in the upper parts of the airways where the mucussecreting goblet cells are located. The mucus catches the invading bacilli, and the cilia on the surface of the cells constantly undulate to move the mucus and trapped foreign particles upward for removal. This system provides the body with an initial physical defense that prevents infection in most persons exposed to tuberculosis [9].

The bacteria that are able to pass the mucociliary system and reach the alveoli are quickly engulfed by alveolar macrophages. This next line of defense is the innate immune system, and it provides an opportunity for the body to destroy the invading mycobacteria and prevent the infection. Many mechanisms and macrophage receptors are involved in uptake of the mycobacteria. The complement system plays a key role in the phagocytosis of the bacteria. The complement protein $\mathrm{C} 3$ binds to the cell wall and enhances recognition of the mycobacteria by macrophages. Opsonization by $\mathrm{C} 3$ is fast, even in the airspaces of a host with no previous exposure to M. tuberculosis [10]. The phagocytosis by macrophages initiates a cascade of events that results in either effective control of the infection (which may be followed by latent tuberculosis) 
or progression to active disease, called primary progressive tuberculosis. The result is mainly determined by the strength of the host defenses and the balance that occurs between host defenses and the invading mycobacteria.

After being swallowed by macrophages, the mycobacteria continue to multiply slowly, with bacterial cell division occurring every 25 to 32 hours. The initial development involves the production of proteolytic enzymes and cytokines by macrophages in order to try to degrade the bacteria. The cytokines that are released attract $\mathrm{T}$ lymphocytes to the site; $\mathrm{T}$ cells now lead the cell-mediated immunity. Macrophages present mycobacterial antigens on their surface to the $\mathrm{T}$ cells. This immune process continues for 2 to 12 weeks; the microorganisms continue to grow until they reach adequate numbers to fully provoke the cell-mediated immune response, which can be detected by a skin test called Tuberculin purified protein derivative (PPD) [11].

For those individuals with intact cell-mediated immunity, the next defensive step is the formation of granulomas around the $M$. tuberculosis organisms. These nodular type lesions, called Ghon complexes, form from an accumulation of activated $\mathrm{T}$ cells and macrophages that limits replication and the spread of the mycobacteria. This destroys the macrophages and produces necrosis at the center of the lesion, yet the bacteria are able to survive since $M$. tuberculosis can change their phenotypic expression in order to enhance survival. By 2 to 3 weeks, the necrotic environment resembles soft cheese, often referred to as caseous necrosis [12]. The conditions for this necrosis include low $\mathrm{pH}$ and limited nutrients. These conditions restrict further growth and the lesions undergo fibrosis and calcification, successfully controlling the infection and causing the bacteria to enter a dormant form.

For immune compromised individuals, granuloma formation is initiated but ultimately is unsuccessful in containing the bacteria. The necrotic tissue undergoes liquefaction and the fibrous wall of the granuloma loses structure. The liquefied necrotic material may then move into a bronchus or nearby blood vessel. If $M$. tuberculosis discharges into a vessel, extra-pulmonary tuberculosis is most likely to occur. Bacilli can also drain into the lymphatic system and collect in the trachea-bronchial lymph nodes of the affected lung, where the organism can form new caseous granulomas [13].

\section{The Bacteriology}

Mycobacterium tuberculosis is a large, non-motile, slow-growing obligate aerobic bacterium. As an obligate aerobe, it has a predilection for the oxygenated environment of the upper lobes of the lungs. $M$. tuberculosis has a doubling time of 18 hours and clinical cultures can take approximately 6-8 weeks. It is resistant to dehydration and so can survive in expectorated sputum. Morphologically the bacterial cell wall contains an array of complex lipids such as mycolic acids, long-chain fatty acids facilitating the acid-fast characteristics; Wax D; and Phosphatides, which contribute to the clinically relevant feature of caseating necrosis [14].

Cord factor, also known as trehalose dimycolate, is a glycolipid found in the cell wall, getting its name from the "serpentine" or cord-like pattern demonstrated by virulent strains [14]. On the surface of M. tuberculosis, cord factor is protective and non-toxic, typically preventing phagocytosis by macrophages during primary infection. In a secondary reaction, cord factor and host lipids in the alveoli may be an initiating factor in caseating necrosis development in lung tissue [15]. Traditionally cord formation has been related to virulence since avirulent $M$. tuberculosis strains do not form cords. However recent findings suggest similar cord formation in non-pathogenic, opportunistic Mycobacterium species, i.e. M. abscessus, M. chubuense, M. gilvum, M. haemophilum, M. marinum, $M$. obuense, $M$. parafortuitum, and $M$. vaccae. The cord patterns among species are not easily distinguished by light microscopy, an important diagnostic implication in minimizing the potential for false negatives and unnecessary exposure to an ineffective and arduous drug regimen [16].

Phthiocerol dimycocerosate, a lipid located in the bacterial cell wall, is also necessary for TB pathogenesis in the lungs. Its early involvement in infection occurs when bacilli encounter host macrophages. Current research suggests that phthiocerol dimycocerosate controls the bacterial invasion of macrophages by targeting lipid organization in the host cell membrane, altering its biophysical properties. These changes in lipid ordering facilitate receptor-mediated phagocytosis of $M$. tuberculosis, contributing to the control of phagosomal $\mathrm{pH}$ and protection of the bacteria from further immune response. The pathogenesis of $M$. tuberculosis hinges on this ability to invade macrophages and use them to evade host cell bactericidal activity [17].

In 1998 the complete gene sequence of $M$. tuberculosis variant (H37Rv) was determined, comprising of $4,411,529$ base pairs and 4000 genes [18]. The genome has an extremely high guanine + cytosine content and is remarkably different than most bacteria in that it possesses dedicated enzymes specialized for lipogenesis and lipolysis. It has been suggested that these fatty acid-utilizing enzymes are potentially associated with the ability of M. tuberculosis to survive in host tissues, using fatty acids as a carbon source [19]. 


\section{Diagnosis}

\section{Culture and Sensitivity}

The permeability of the aforementioned barrier enables the bacterium to resist conventional gram staining, causing gram stains to show a weak positive, or to show up white; so typically an alternative (acidfast) stain is used instead. Acid-fast, also known as the Ziehl-Neelsen stain refers to the ability of $M$. tuberculosis to retain carbolfuchsin stain, despite decolorization treatment with ethanol-hydrochloric acid [20]. Preparation prior to staining involves $\mathrm{NaOH}$ treatment, which destroys unwanted bacteria, human cells, and fluid, followed by centrifugation. This is followed by culture on Lowenstein-Jensen media for up to 8 weeks. Lowenstein-Jensen media contains complex nutrients and dyes, i.e. egg yolk and malachite green dyes; dyes inhibit normal flora present in sputum samples [14].

Clinically, tuberculosis can be diagnosed by signs and symptoms, characteristics on chest radiography, and positive skin reactivity findings from the tuberculin (Mantoux) skin test $[21,22]$. Sign and symptoms suggestive of TB include: significant cough that lasts 3 weeks or longer, chest pain, hemoptysis, coughing up sputum (productive cough), fatigue, weight loss, anorexia, chills, pyrexia, night sweats [23]. On chest radiograph, TB disease activity is evidenced by any parenchymal, nodal, or pleural abnormality with or without associated calcification [24]. Confirmation of these findings and tests are supported by the microscopic identification of acid-fast rods and the culture of the bacteria.

A second approach, known as interferon-gamma release assay (IGRA) measures the body's immune reaction to TB by testing blood in the laboratory [21]. More specifically the level of interferon-gamma is measured upon exposure of blood cells to an antigen from $M$. tuberculosis. A positive test indicates infection with TB; however additional tests are required to determine whether a latent infection or active TB disease is present. A negative test showing a lack of reaction indicates a lower likelihood of TB infections or disease. This test is not influenced by BCG immunization. Laboratory diagnoses may also be obtained within 2 weeks by detecting radioactive carbon dioxide using a liquid BACTEC medium. If growth occurs, further biochemical tests can be performed, including niacin detection, which is almost exclusively seen in $M$. tuberculosis [14].

During treatment, it may be necessary to determine the presence of drug resistance. Detection of mutations in the catalase gene confirms resistance to isoniazid and RNA polymerase gene mutations confirm resistance to rifampin. The luciferase assay can expediently detect drug-resistant organisms as well [14].

\section{Treatment and Prognosis}

Patients with latent tuberculosis infection have the bacteria in their bodies but do not typically present with symptoms because the bacteria are not active. If the bacteria become active and multiply, then the symptoms of TB will become evident in the patient. For this reason, patients with known latent $\mathrm{TB}$ are prescribed preventative pharmacological interventions. The current medications that are used for the treatment of latent tuberculosis are isoniazid, rifampin, and rifapentine [25].

Non-resistant TB is usually treated by a regimen of several drugs taken for a period of 6 to 9 months. Currently, there are 10 drugs that are approved by the FDA for the treatment of active TB. Of these approved drugs, the first-line pharmacological intervention that forms the core treatment regimen includes isoniazid, rifampin, ethambutol, and pyrazinamide [25].

Treatment regimens for non-resistant TB have an initial phase of 2 months, followed by a continuation phase of usually 4-7 months. The 6-month regimen consists of isoniazid, rifampin, and pyrazinamide given for 2 months followed by isoniazid and rifampin for 4 months. Ethambutol or streptomycin is added in the first 2 months in patients with advanced disease. The success rate with the 6-month regimen in sputum conversion (conversion defined as a negative culture in 3 consecutive samples taken 1 day apart) is far beyond $90 \%$ within the first two months of therapy. The relapse rate after 3-5 years is about $0-3 \%$ [26]. It is very important to complete the treatment regimen because bacteria could still be active and become resistant to these first-line drugs if the treatment is stopped prematurely. For this reason, physician supervision and follow-up become important to ensure patient compliance [27].

Shortening anti-tuberculosis treatment regimens is one strategy expected to improve patient adherence to treatment, resulting in better case management and disease control and minimizing the risk of drug resistance. Gatifloxacin, normally a second-line drug, was selected for a recent phase 3 trial to evaluate the efficacy of a 4-month regimen compared to the standard 6 months. This trial assessed the effect of shortening rifampin-sensitive TB treatment by using a fluoroquinolone-based approach. The results of the study failed to show that 4-month treatment with gatifloxacin, which was substituted for ethambutol, was non-inferior to the standard 6-month regimen. There was a higher recurrence rate observed with the 4month regimen [28]. 
MDR-TB and XDR-TB are inherently more difficult to treat because the treatment must be individualized and closely monitored. Depending on the susceptibility of the infection, treatment regimens for resistant strains can last up to 36 months or more. An additional complication of this treatment is that, while first-line TB drugs are relatively nontoxic, second-line treatments like fluoroquinolones carry the risk of more serious side effects. Second-line treatments also tend to be more expensive than first-line drugs, and they may not be available in areas where access to health care is limited. These factors all complicate the treatment of an illness that is already difficult to defeat [29].

An important strategy to address MDR-TB and XDR$\mathrm{TB}$ is the development of novel pharmacological approaches to circumvent bacterial resistance. These research foci vary widely, as does their effectiveness. One recent study examined the addition of efflux inhibitors including verapamil, chlorpromazine, farnezol, reserpine, and others, as adjuvants to increase the effectiveness of antibiotic regimens already used in the treatment of TB. The efflux inhibitors reduce the bacterium's ability to expel the antibiotic before it achieves its intended effect. So while efflux inhibitors are not themselves toxic to these microorganisms, their use in a TB regimen may prolong the intracellular presence and subsequent damage of the concurrentlyadministered antibiotics [30].

Other approaches currently being explored include the re-purposing of drugs currently in use for other conditions, in which anti-TB activity has been observed. Some of these drugs are not currently in use as antibiotics at all. One example of this is the antipsychotic thioridazine, a member of the phenothiazine class of neuroleptics which exhibits anti-mycobacterial action and has been shown to be effective in the treatment of some XDR-TB cases. Trials for the use of thioridazine in this capacity are expected to begin soon [29].

There are also a few new drugs in development with novel mechanisms of action against TB. These include bedaquiline, a diarylquinoline which inhibits ATP synthesis in mycobacteria, and delamanid, which inhibits bacterial mycolic acid (an important cell-wall component) synthesis. As these drugs and others proceed through trials, their efficacy and safety for patients will become more evident [31].

\section{Epidemiology}

\section{Current Global Distribution}

The latest trends in the global distribution of tuberculosis were published in 2018 by the World Health Organization in their annual report on tuberculosis [32]. Globally, TB is one of the top 10 causes of death and the leading cause of death in HIV infection / AIDS. Many people continue to fall sick each year from TB infection. TB caused an estimated 1.3 million deaths among HIV-negative individuals and an estimated 300000 death among HIV-positive individuals in 2017. Worldwide, an estimated 10.0 million people developed TB disease in 2017 with a breakdown of 5.8 million men, 3.2 million women and 1.0 million children. Cases were reported in all countries and age groups; overall $90 \%$ were adults (aged $\geq 15$ years), 9\% were individuals living with HIV (72\% in Africa) and two thirds were from eight countries: India (27\%), China (9\%), Indonesia (8\%), the Philippines (6\%), Pakistan (5\%), Nigeria (4\%), Bangladesh (4\%) and South Africa (3\%). The listed 8 countries and 22 other countries in WHO's list of 30 high TB burden countries made up for $87 \%$ of the world's cases; while $6 \%$ of global cases were in the WHO European Region (3\%) and WHO Region of the Americas (3\%) [32].

In 2017, fewer than 10 new cases per 100000 individuals were reported in most high-income countries, $150-400$ in the majority of the 30 high TB burden countries, and over 500 in a few countries including Mozambique, the Philippines and South Africa [32].

Globally in 2017, an estimated 558000 individuals developed TB that had resistant to rifampicin (RR-TB), considered to be the most effective first-line drug, and out of these, $82 \%$ had multidrug-resistant TB (MDR-TB). India (24\%), China (13\%) and the Russian Federation $(10 \%)$ accounted for almost half of the world's cases of MDR/RR-TB [32].

Globally prevalence of MDR/RR-TB in new TB cases is $3.5 \%$ while that of previously treated cases is $18 \%$ with the highest proportions ( $>50 \%$ in previously treated cases) found in countries of the former Soviet Union. In 2017, among cases of MDR-TB, 8.5\% were estimated to have extensively drug-resistant TB (XDRTB) [32].

Latent TB infection is estimated to be present globally in about 1.7 billion people $(23 \%$ of the world's population). These individuals are at risk of developing active TB disease during their lifetime [32].

TB incidence rate is falling globally at about $2 \%$ per year. The fastest regional declines from 2013 to 2017 were reported in the WHO European Region (5\% per year) and the WHO African Region (4\% per year). Similarly, TB deaths among HIV-negative individual has fallen globally by an estimated 29\% since the year 2000 while that of the HIV-positive individual has also fallen by $44 \%$ since the same year. Among WHO regions, the 
fastest declines in the 5 years 2013-2017 were reported in the WHO European Region (11\% per year) and the WHO South-East Asia Region (4\% per year) [32].

\section{Vulnerable Populations}

Some populations are particularly vulnerable to tuberculosis infection. These populations are comprised of HIV-positive patients and other immunosuppressed patients including alcoholics and intravenous drug users, as well as people in very crowded living conditions such as prisoners or those in densely populated urban areas [33]. People from countries with a high incidence \& prevalence of tuberculosis, especially those living in poverty, are also more susceptible. In developed countries, immigrants from areas with a high TB prevalence are more vulnerable than the general population.

Children are a statistically significant portion of these vulnerable populations because their symptoms may go undetected and they may suffer for a long time before finally being diagnosed and treated [34]. Many tuberculosis control programs have neglected this population even while faced with a rising number of infections. Children have a higher rate of primary disease progression after infection, which means they may spread the infection faster than other patients. International TB control units may not be accounting for the increase in incidence among juveniles, and thus disregarding an important vector for the spread of infection [35].

TB infection is very common among alcoholics and intravenous drug users, especially those infected with HIV. These patients may be unwilling or unable to get timely treatment for symptoms, so their infections may go undetected until they are severe and difficult to treat. Preventative care and supervision are important factors in controlling TB in these patients [36].

Another worrying vulnerability in HIV-positive patients is the possibility of reactivation of latent TB infection. As mentioned previously, TB infection can sometimes persist in a latent, non-pathogenic state. Corbett and associates have shown a pattern in which the presence of HIV in these patients actually awakens the latent TB infection and they become likely to develop active TB. Globally, tuberculosis is the leading cause of death of HIV positive patients [37]. Residents of urban areas, where there is an estimated incidence of infection up to 3-5 times higher than in rural areas, are also vulnerable. This may be due to the fact that people in urban areas often live in densely packed conditions and are in contact with a large number of people, and thus more likely to be exposed to pathogens [38]. Additionally, an overall decrease in baseline health and an increase in respiratory complaints and asthma due to pollution and other factors may prevent these patients from seeking care in a timely manner after the onset of symptoms [39]. These factors also apply to other conditions of the dense population, including prisons and shelters. In order to decrease the rate of infection in this population, control units need to aim at increasing the awareness of TB, its signs and symptoms, and the importance of care across urban and other densely populated areas [40].

An interesting, perhaps somewhat counterintuitive fact is the phenomenon of genetic resistance to TB infection found in some urban populations. There is evidence that since the onset of urbanization in ancient times, the increased prevalence of TB in crowded conditions led to the development of genetic resistance in some families, particularly those who continued to reside in these areas for many generations. Research by the University of London and the University of Oxford determined the frequency of an allele (SLC11A1 1729+ $55 \mathrm{del} 4$ ) associated with a natural resistance to TB and Leprosy [41]. This allele showed a significant correlation with the long-term urban settlement. This resistance is likely due to natural selection, especially considering the astronomical mortality rate of TB before the development of antibiotics in the 20th century. This is good news for an ever more denselypopulated world, as the persistence of this resistant population may eventually increase the resistance of the general population to TB over time.

Most vulnerable populations have in common that they are lower on the socio-economic spectrum. In fact, there is a clear correlation between poverty and vulnerability to TB infection. Ignorance or denial of the significance of symptoms, delayed or inaccurate diagnosis and lack of access to care and treatment are all factors that contribute to this vulnerability [42]. Addressing these issues through targeted campaigns to increase patients' awareness, access to accurate diagnostic ability and effective treatment should be the strategies for success of TB control units worldwide.

\section{Global Initiatives}

While tuberculosis infection spans the globe, the fight against it has been spearheaded by a few key organizations. The WHO has implemented far-reaching programs that work with local governments, other international aid organizations, NGOs, and other stakeholders to develop research and provide equipment and services to improve community management of this epidemic. In 1995, the WHO first began to standardize the collection of regular reports of global TB incidence and other statistics. The year 2015 marked the twentieth year of TB data collection, and the fifteenth year since the adoption of Millennial Development Goals (MDGs) signed by all 191 United 
Nations member states in 2000. These goals proposed specific metrics to track progress on a variety of issues, including reducing the incidence and improving treatment of infectious diseases. One of the declared MDGs was to reverse the then-increasing incidence of TB, a goal which has been met by its evaluation date of 2015. Another was to increase the TB cure rate, which has risen from less than $80 \%$ in 1990 to around $86 \%$ since 2013. However, as the time period encompassed by the MDGs comes to an end in 2015, the global fight against TB is far from over [2].

For more than two decades, the WHO's anti-TB efforts have been based on the Directly-Observed Treatment, Short Course or "DOTS" treatment strategy as the global standard of care. This strategy includes five elements necessary for effective disease management, described in Figure 1 below.

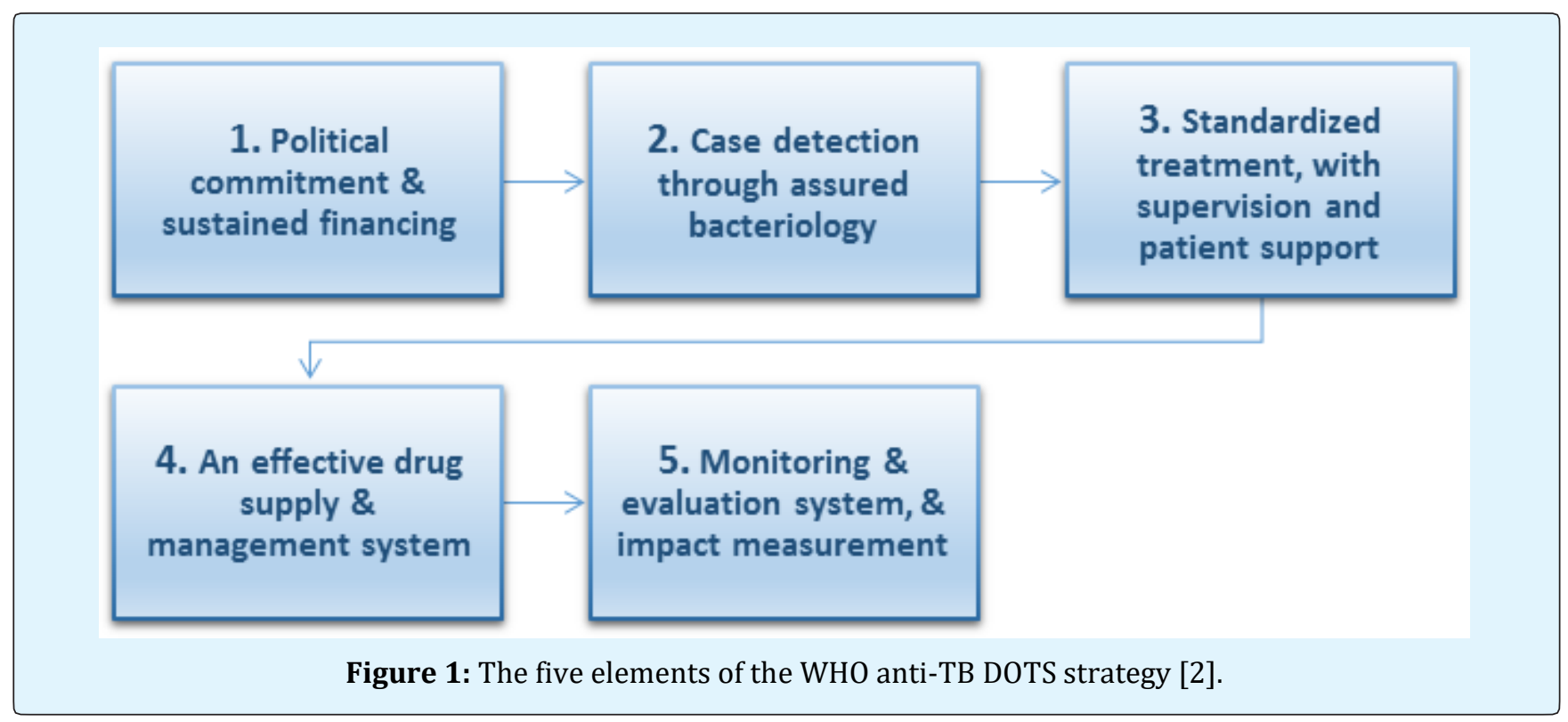

The DOTS principles illustrate the many facets of the struggle to control infectious diseases. This system has provided a basis for global TB care, including the WHO "Stop TB" program, initiated in 2006, and it continues to guide the "End TB" program that began in 2015. Clearly, difficulties in any one element of this strategy may compromise the success of a control program as a whole. Given the myriad uncertainties in many areas of endemic TB, it is easy to see how challenging this effort really is. Despite this, the WHO has set ambitious goals and progress metrics to guide its many programs for the next two decades (Table 1) [2].

\begin{tabular}{|c|c|c|c|c|}
\hline WHO Strategic Goals: & \multicolumn{2}{|c|}{ Milestones } & SDGs* & End TB Goals \\
\hline Year & $\mathbf{2 0 2 0}$ & $\mathbf{2 0 2 5}$ & $\mathbf{2 0 3 0}$ & $\mathbf{2 0 3 5}$ \\
\hline Decrease Incidence & $20 \%$ & $50 \%$ & $80 \%$ & $90 \%$ \\
\hline Decrease Deaths & $35 \%$ & $75 \%$ & $90 \%$ & $95 \%$ \\
\hline
\end{tabular}

Table 1: WHO strategic goals, 2015-2035 [2].

*SDGs- Sustainable Development Goals

In order to achieve the End TB Strategy milestones for 2020 and 2025, TB diagnosis, treatment and prevention services should be provided within the context of progress towards universal health coverage (UHC), as well as the presence of a multisectoral action to address the social and economic factors that drive TB epidemics [32]. SDG Target 3.8 is aimed to achieve UHC by 2030. WHO estimation published in 2017 believed that most middle-income countries could mobilize the funding required to achieve UHC by 2030 from domestic resources, while this is not likely to be achieved in lowincome countries [32].

\section{Antibiotic Resistance}

One of the biggest obstacles in the global fight against tuberculosis is bacterial development of resistance to the essential drugs used to treat it. After centuries of rampant pathogenesis and devastating mortality rates, a series of incredible discoveries in the 20th century led to the development of effective treatments for this formerly invulnerable infection. However, over time, as these treatments were massively and sometimes inappropriately administered, and unsupervised patients failed to rigorously complete their prescribed regimens, the highly versatile $M$. 
tuberculosis developed a variety of mutations to subvert the cellular mechanisms of these drugs. Treatment of MDR-TB and XDR-TB takes significantly longer (up to 36 months or more) than treatment of non-resistant TB, and the second-line and third-line drugs required are typically more expensive and far more toxic than firstline drugs. These factors may preclude patient compliance without financial assistance and vigilant supervision and follow-up by healthcare professionals. This has led to the persistence of $\mathrm{TB}$ as the most common cause of death from infectious disease in the world and continues to challenge healthcare practitioners globally every day [1].

Multiple-drug resistant strains of tuberculosis are resistant to both of the most commonly used first-line drugs, isoniazid, and rifampin. These drugs have been in use for over 50 years and their mechanisms of action have been extensively studied. This has enabled the elucidation of $M$. tuberculosis' ability to evade them through genetic mutation. As the cellular mechanisms of TB drugs differ, so do the bacterial mutations that confer resistance to these drugs.

It is well known that the catalase-peroxidase enzyme, encoded by the katG gene of $M$. tuberculosis, is responsible for activating the pro-drug isoniazid. The activation of isoniazid leads to an isonicotinoyl acyl radical that then combines with $\mathrm{NAD+} / \mathrm{NADH}$ to form the isoniazid-NADH adduct (INH-NADH) [43-45]. The isoniazid-NADH adduct is a potent inhibitor of inhA, a gene which produces an enoyl acyl-carrier protein reductase involved in the production of mycolic acids, which are the main structural components of the mycobacterial cell wall [46-48]. Mutations in katG can lead to isoniazid-resistance due to their inability to activate the isoniazid prodrug, preventing its effect [4951].

In contrast, rifampin acts by inhibiting bacterial DNA-dependent RNA synthesis. It accomplishes this by inhibiting bacterial DNA-dependent RNA polymerase. Rifampin binds to RNA polymerase at a site adjacent to the RNA polymerase active center and blocks RNA synthesis by physically blocking the formation of the phosphodiester bond in the RNA backbone, preventing extension of RNA products beyond a length of 2-3 nucleotides [52,53]. Resistance to rifampicin arises from mutations that alter residues of the rifampin binding site on RNA polymerase, resulting in decreased affinity for rifampin and prevention of its effect [53].

Extensive drug resistant (XDR-TB) strains of bacteria were first reported in 2006. In addition to isoniazid and rifampicin, XDR strains show resistance to any of the fluoroquinolones (e.g. ofloxacin, moxifloxacin, and others) and to at least one of three injectable second-line drugs (amikacin, capreomycin or kanamycin). Extensive drug-resistant tuberculosis cases are rare; however, 77 countries worldwide had reported at least one case by the end of 2011. Information from countries with reliable data suggests that approximately $9.7 \%$ of MDR-TB cases worldwide have XDR tuberculosis [54].

Not all of the cellular mechanisms of extensive drug resistance have been described. However, the drugs involved have been in use in various capacities for some time, so some insights into bacterial resistance have been observed. Fluoroquinolones are derivatives of nalidixic acid, a substance that was discovered as a byproduct of an antimalarial compound. This acid's antibiotic effects were first observed in 1965, but its action against TB was not evident until much later. Derivatives of this acid interfere with bacterial DNA replication by inhibiting a protein called topoisomerase II (also called DNA gyrase), which is responsible for initiating super coiling of the cell's DNA. A similar protein, topoisomerase IV, is also inhibited by these drugs, but only type II is found in M. tuberculosis. Resistance to fluoroquinolones occurs when mutations develop in bacterial genes called gyrA and gyrB, which are responsible for the production of this protein $[55,56]$.

The injectable second-line drugs kanamycin and amikacin both inhibit bacterial protein synthesis at 16sRNA. Bacterial mutations in the rrs and eis genes have been shown to confer resistance to kanamycin, but only rrs mutations show evidence of resistance to amikacin. In contrast, capreomycin, another injectable, is able to bind to bacterial ribosomes at the interface between small and large ribosomal subunits. Resistance to capreomycin is conferred by mutations at both rrs and eis, as well as an additional location called tlyA $[55,56]$.

Resistance to other common second-line TB drugs including ethionamide, cycloserine, macrolides like clarithromycin, and others has been observed as well. In 2009 , Velayati et al. reported an observation of patients in Iran with XDR-TB whose infections were also resistant to all second-line drugs available at the time. The authors called this new level of resistance totallydrug resistant, or TDR-TB. Cultures of M. tuberculosis from these patients were not identical, however. The bacterial strains cultured from these individuals were found to originate from at least four different known MDR-TB superfamilies. This was not the detection of an entirely new, super-resistant strain of bacteria. Instead, it was evident that these patients had been intermittently and ineffectively treated for less resistant TB infection prior to developing this extreme level of drug resistance. If these patients had been treated appropriately for their original TB infections, it is unlikely that any further drug resistance would have 


\section{Epidemiology International Journal}

developed [57]. This report was not the first time TB patients with extreme resistance were reported. In 2007 , two patients in Italy were found to be resistant to all available first-line and second-line drugs available. The authors reporting those cases called the new level of resistance XXDR, or extremely-drug resistant TB. There is as yet no official nomenclature or standard definition of any higher resistance than XDR-TB, but if treatment inconsistencies continue in areas where MDR-TB and XDR-TB are already endemic, then cases like this may become more common [58].

The WHO estimates that there were around 480,000 MDR-TB cases worldwide in 2014. Unfortunately, only a small proportion of these cases are detected and treated appropriately. This is due to a variety of factors, including lack of diagnostic equipment or training, lack of access to appropriate treatment, and lack of patient compliance. Another, perhaps more worrying factor is inconsistencies in the training of physicians in endemic TB areas. Reports on TDR-TB patients in India in 2012 confirmed that these patients had been treated for TB by an average of four different physicians over a period of 18 months, and that the treatments they received were inconsistent and often inappropriate. This shows that standardizing effective treatment protocols is only one part of the solution to resistance; physician education, especially in high-burden MDR-TB and XDRTB areas, is an essential part of this as well. Timely detection and appropriate treatment of resistant TB strains is extremely important to prevent further evolution and spread of "untreatable" TDR-TB strains [59].

\section{Prevention and Control}

The major health-care interventions for preventing new infections and progression to TB disease are the treatment of latent TB infection and vaccination of children with the bacille Calmette-Guérin (BCG) vaccine. While preventive treatment for a latent TB infection is expanding, accessibility of care is still a problem to those that require it; this is unlike BCG vaccination coverage which is high [32]. WHO has strongly recommended treatment for latent TB infection in two important groups' i.e. individuals living with HIV, and children aged less than 5 years who are household contacts of an individual with bacteriological confirmed pulmonary TB [32].

Progress is being made in the fight against TB infection on many fronts. One important strategy to control this disease is the development of tools to provide a quick, accurate diagnosis of drug-resistant strains of bacteria in the field. Another, equally critical element is coordination between healthcare providers. Infrastructure, up-to-date hospitals and clinics, and community education all play essential roles in the struggle to control this disease [2].

A serious concern in modern TB treatment is the delay in diagnosis of drug-resistant strains of the bacteria, which require specialized treatment regimens. This is especially important because the areas where MDR-TB and XDR-TB are endemic are also largely underdeveloped areas with limited access to modern healthcare and laboratory equipment. In cases where TB is suspected but the bacteria's susceptibility is unknown, patients may receive ineffective treatment before the resistance of their infection is identified. This can increase the patient's risk of morbidity and mortality from TB, as well as encourage bacterial development of more extensive resistance mechanisms to a broader range of antibiotics. This delay may also prolong the window for transmission of the infection, perpetuating the reservoir of resistant microorganisms. It is clear that access to timely diagnosis is essential to providing appropriate treatment and reducing the prevalence of resistant organisms [60].

Currently, a widely-used standard for drug susceptibility testing (DST) of TB is the BACTEC MGIT960, a fully-automated system created for the culture and identification of Mycobacterial strains through DNA analysis which has been in use since 1998. In a study by Catanzaro and associates, the BACTEC MGIT960 was compared with the three rapid diagnostic kits for performance and accuracy. The study included the Line Probe Assay (LPA) and Pyrosequencing (PSQ), which both produced results in an average of 1.1 days, and Microscopic Observation of Drug Susceptibility (MODS), which produced results in an average of 14.3 days. In contrast, the BACTEC MGIT960 took an average of 24.7 days to produce results. All three rapid test kits had very high specificity for detection of the most common types of drug resistance in MDR-TB and XDR$\mathrm{TB}$, ranging from $97-100 \%$. The sensitivity of the kits was somewhat lower but still significantly high: for resistance to isoniazid, rifampin, moxifloxacin, and ofloxacin, sensitivity was found to be $94-100 \%$, for amikacin and capreomycin, it was $84-90 \%$, and for kanamycin, it was $48-62 \%$. This means that all three rapid test kits were able to identify bacterial strains with 6 out of the 7 most common types of drug resistance in almost $100 \%$ of patients, in a fraction of the time necessary for other methods [60].

Due to a lack of data on the effectiveness and accuracy of these rapid test kits, only one of them (a version of the Line Probe Assay called MTBDRplus) is currently WHO-approved and has been in use since 2008. Increasing the financial and human resources necessary to obtain and analyze data on the use of this technology could have a hugely positive impact on the timely diagnosis of MDR-TB and XDR-TB [60]. 
Another problematic aspect of diagnosis in the field is the storage and transport of samples to testing locations. A recent study evaluated the safety and efficiency of four different storage techniques in locations where samples have to be transported (or even mailed) to labs where they can be analyzed. The labs involved were in Madagascar, India, Argentina, and Brazil, all relatively high-burden countries with challenging environments. The four storage methods were traditional Ziehl-Neelsen (ZN) slides, storage of samples in ethanol, and two commercially-produced card systems called the FTA card and the GenoCard. All four methods were tested with susceptible $M$. tuberculosis strains and MDR-TB strains, as well as with non-tubercular Mycobacterial strains for reference. The four methods all showed excellent results; 100\% specificity and $100 \%$ sensitivity at 3 out of the 4 participating labs, and $88-100 \%$ specificity and $80-84 \%$ sensitivity at the fourth lab. Out of 200 samples tested with each method i.e. 100 susceptible TB and 100 MDRTB, no less than 194 samples were correctly identified by any method. The FTA card resulted in 200 correctly identified samples, ethanol storage resulted in 197 correct identifications, the ZN slides produced 195 correct results, and the GenoCard resulted in 194 samples being correctly identified. These results are hugely positive for areas where one or more storage method may be impractical or unavailable. However, this study, which tested the efficacy of these methods using isolated Mycobacterial strains, was only the beginning; more testing will have to be performed using sputum samples before these methods can become accepted protocols [61].

Another important strategy to control TB infection is an investment in the structure and design of hospitals and community healthcare centers in areas of endemic XDR-TB. In many underdeveloped areas, hospitals are crowded, sanitation is subpar, and the structure of buildings may prevent the necessary level of isolation of contagious patients. This is especially imperative in areas with a high prevalence of HIV and AIDS, as these patients are particularly vulnerable to the pathogenesis of TB. Nosocomial spread of extremely virulent XDR-TB can be devastating, as seen in Tugela Ferry, South Africa in 2005 and 2006 [62].

Community and patient awareness of TB and the implications of antibiotic resistance are also important for controlling this disease. Globally, one of the most active XDR-TB endemic areas is found in the underdeveloped regions of Eastern Europe [63]. A recent survey of the relevant knowledge of school and other institutional staff in the Republic of Georgia revealed a large amount of misinformation about antibiotics. $55 \%$ of respondents believed that antibiotics were effective against both bacterial and viral infections, and $55 \%$ believed that antibiotics would improve the common cold [64]. This is concerning since antibiotics are not regulated by prescription in Georgia; instead, they are available over the counter for anyone to purchase at any time. 91\% of survey respondents had used antibiotics, but their other responses show that this use is likely inappropriate in around $55 \%$ of cases. Such rampantly inappropriate use of antibiotics has certainly contributed to the development and prevalence of XDR-TB in this region. This illustrates how important it is for TB control programs to increase awareness of these issues, especially in MDR-TB and XDR-TB endemic areas [64].

In order to effectively implement these principles, investments are necessary for equipment, staff, community outreach, and transport and delivery of supplies. Yet anti-TB efforts continue to be underfunded in comparison to less prevalent diseases such as HIV. In 2014, TB caused 1.5 million deaths, whereas HIV caused around 1.2 million. This difference in mortality rates is not especially large, but according to the WHO, in 2014 only 6.6 billion dollars were invested in the fight against TB, whereas investment in HIV programs worldwide was nearly 20.2 billion dollars. This huge disparity in funding prevents effective implementation of programs that have been proven to be successful for TB control. Clearly, the lack of funding is directly related to the continued prevalence of TB [2].

\section{Progress in Research and Development}

Breakthroughs in technology are required to accelerate the annual decline in the global TB incidence rate to an average of $17 \%$ per year. Area of research focus include a vaccine to lower the risk of infection, a vaccine or new drug treatment to reduce the risk in latently infected people, rapid diagnostics for use at the point of care and simpler, shorter drug regimens for treatment. There is slow progress in the development pipelines with few diagnostic technologies emerging in 2017 in spite of recent increase funding for TB research and development. Presently under clinical trials are 20 drugs, several treatment regimens and 12 vaccine candidates [32].

\section{Conclusion}

Despite vast improvements in research and technology and the development of multiple drug regimens to battle this insidious killer, $M$. tuberculosis continues to be a major health concern worldwide. As TB infection continues to be the most prevalent fatal infectious disease in the world, funding for research and program implementation has trailed global investment in other diseases. Advances in diagnostic tools, active patient supervision, and a global focus in detecting and mapping strains of drug-resistant pathogens are proven strategies for controlling this disease. However, integral 


\section{Epidemiology International Journal}

to these clinically important strategies is a solid commitment by the international community to finally eradicate this devastating infection.

\section{References}

1. World Health Organization (2010) Multidrug and extensively drug-resistant TB (M (No. WHO/HTM/TB/2010.3). Geneva: World Health Organization.

2. World Health Organization (2015) Global tuberculosis report 2015, 20th ed. World Health Organization.

3. Frith J (2014) History of tuberculosis. Part 1phthisis, consumption and the white plague. J Military Veterans Health 22(2): 29.

4. Cambau E, Drancourt M (2014) Steps towards the discovery of Mycobacterium tuberculosis by Robert Koch, 1882. Clin Microbiol Infect 20(3): 196-201.

5. Daniel TM (2006) The history of tuberculosis. Respir Med 100(11): 1862-1870.

6. Fox W, Ellard GA, Mitchison DA (1999) Studies on the treatment of tuberculosis undertaken by the British Medical Research Council tuberculosis units, 1946-1986, with relevant subsequent publications. Int J Tubercul Lung Dis 3(10): S231-S279.

7. Keshavjee S, Farmer PE (2012) Tuberculosis, drug resistance, and the history of modern medicine. $\mathrm{N}$ Engl J Med 367(10): 931-936.

8. Cegielski JP (2010) Extensively drug-resistant tuberculosis: "there must be some kind of way out of here". Clin Infect Dis 50(3): S195-S200.

9. Frieden TR, Sterling TR, Munsiff SS, Watt CJ, Dye C (2003) Tuberculosis. Lancet 362(9387): 887-899.

10. Van Crevel R, Ottenhoff TH, van der Meer JW (2002) Innate immunity to Mycobacterium tuberculosis. Clin Microbiol Rev 15(2): 294-309.

11. Dheda K, Booth H, Huggett JF, Johnson MA, Zumla A, et al. (2005) Lung remodeling in pulmonary tuberculosis. J Inf Dis 192(7): 1201-1209.

12. Du Toit LC, Pillay V, Danckwerts MP (2006) Tuberculosis chemotherapy: current drug delivery approaches. Respir Res 7(1): 118.

13. Knechel NA (2009) Tuberculosis: pathophysiology, clinical features, and diagnosis. Critical Care Nurse 29(2): 34-43.
14. Levinson W (2010) Review of medical microbiology and immunology. The McGraw-Hill Companies.

15. Hunter RL, Olsen MR, Jagannath C, Actor JK (2006) Multiple roles of cord factor in the pathogenesis of primary, secondary, and cavitary tuberculosis, including a revised description of the pathology of secondary disease. Ann Clin Lab Sci 36(4): 371-386.

16. Julián E, Roldán $M$, Sánchez-Chardi $A$, Astola $O$, Agustí G, et al. (2010) Microscopic cords, a virulence-related characteristic of Mycobacterium tuberculosis, are also present in nonpathogenic mycobacteria. J Bacteriol 192(7): 1751-1760.

17. Astarie-Dequeker C, Le Guyader L, Malaga W, Seaphanh FK, Chalut C, et al. (2009) Phthiocerol dimycocerosates of $M$. tuberculosis participate in macrophage invasion by inducing changes in the organization of plasma membrane lipids. PLoS Pathog 5(2): e1000289.

18. Cole S, Brosch R, Parkhill J, Garnier T, Churcher C, et al. (1998) Deciphering the biology of Mycobacterium tuberculosis from the complete genome sequence. Nature 393(6685): 537-544.

19. Smith I (2003) Mycobacterium tuberculosis pathogenesis and molecular determinants of virulence. Clin Microbiol Rev 16(3): 463-496.

20. Nikaido H, Brennan PJ (1995) The envelope of mycobacteria. Annu Rev Biochem 64: 29-63.

21. CDC. Tuberculosis (TB) Testing \& Diagnosis.

22. Goering R, Dockrell H, Zuckerman M, Roitt I, Chiodini PL (2012) Mims' medical microbiology. Elsevier Health Sciences.

23. CDC. Tuberculosis (TB) Disease: Symptoms \& Risk Factors.

24. Leung AN (1999) Pulmonary tuberculosis: the essentials. Radiol 210(2): 307-322.

25. CDC Multidrug-Resistant Tuberculosis (MDR TB).

26. Fischer B, Ferlinz R (1995) Therapy and prognosis of tuberculosis. Versicherungsmedizin 47(6): 212216.

27. Brode SK, Varadi R, McNamee J, Malek N, Stewart S, et al. (2015) Multidrug-resistant tuberculosis: Treatment and outcomes of 93 patients. Can Respir J 22(2): 97-102.

28. Merle CS, Fielding K, Sow OB, Gninafon M, Lo MB, et al. (2014) A four-month gatifloxacin-containing 
regimen for treating tuberculosis. New Eng J Med 371(17): 1588-1598.

29. Maitra A, Bates S, Kolvekar T, Devarajan PV, Guzman JD, et al. (2015) Repurposing-a ray of hope in tackling extensively drug resistance in tuberculosis. Int J Infect Dis 32: 50-55.

30. Coelho T, Machado D, Couto I, Maschmann R, Ramos D, et al. (2015) Enhancement of antibiotic activity by efflux inhibitors against multidrug resistant Mycobacterium tuberculosis clinical isolates from Brazil. Frontiers Microbiol 6: 330.

31. Chakraverty R, Debnath T, Ghosh A (2015) Emerging therapeutic strategies for combating drug resistance in tuberculosis: An appraisal. Creat J Pharmac Res 1(3): 108-116.

32. World Health Organization (2018) Executive Summary; WHO Global Tuberculosis Report 2018.

33. Yamamura $M$, Santos-Neto $M$, Santos $R$, Garcia $M$, Nogueira J, et al. (2015) Epidemiological characteristics of cases of death from tuberculosis and vulnerable territories. Revista LatinoAmericana de Enfermagem 23(5): 910-918.

34. Ross J, Cattamanchi A, Miller C, Tatem A, Katamba $A$, et al. (2015) Investigating Barriers to Tuberculosis Evaluation in Uganda Using Geographic Information Systems. Ame J Tropical Med Hygiene 93(4): 733-738.

35. Stoesslé P, González-Salazar F, Santos-Guzmán J, Sánchez-González N (2015) Risk factors and current health-seeking patterns of migrants in northeastern Mexico: healthcare needs for a socially vulnerable population. Frontiers Public Health 3: 191.

36. Klinkenberg E, Manissero D, Semenza JC, Verver S (2009) Migrant tuberculosis screening in the EU/EEA: yield, coverage and limitations. Eur Respir J 34(5): 1180-1189.

37. Corbett EL, Watt CJ, Walker N, Maher D, Williams $B G$, et al. (2003) The growing burden of tuberculosis: global trends and interactions with the HIV epidemic. Archiv Inter Med 163(9): 10091021.

38. Abe T, Iinuma Y, Ando M, Yokoyama T, Yamamoto T, et al. (2003) NRAMP1 polymorphisms, susceptibility and clinical features of tuberculosis. J Infec 46(4): 215-220.

39. Potter JL, Inamdar L, Okereke E, Collinson S, Dukes $\mathrm{R}$, et al. (2015) Support of vulnerable patients throughout TB treatment in the UK. J Public Health 38(2): 391-395.

40. Mulder C, Klinkenberg E, Manissero D (2009) Effectiveness of tuberculosis contact tracing among migrants and the foreign-born population. Euro Surveill 14(11): 19153.

41. Hassan F (2000) The origins and development of African livestock: archaeology, genetics, linguistics and ethnography. Routledge, Abingdon, Oxford, UK.

42. RIT J (2016) Tuberculosis Annual Report 2014--(1) Summary of Statistics on Tuberculosis Notification and Foreign-born Tuberculosis Patients. Kekkaku 91(2): 83-90.

43. Scior T, Meneses Morales I, Garcés Eisele SJ, Domeyer D, Laufer S (2002) Antitubercular isoniazid and drug resistance of Mycobacterium tuberculosis-a review. Arch Pharm: Int J Pharm Med Chem 335(11-12): 511-525.

44. Vilcheze C, Jacobs Jr WR (2007) The mechanism of isoniazid killing: clarity through the scope of genetics. Annu Rev Microbiol 61: 35-50.

45. Slayden RA, Barry CE (2000) The genetics and biochemistry of isoniazid resistance in Mycobacterium tuberculosis. Microbes Infection 2(6): 659-669.

46. Rozwarski DA, Grant GA, Barton DH, Jacobs WR, Sacchettini JC (1998) Modification of the NADH of the isoniazid target (InhA) from Mycobacterium tuberculosis. Sci 279(5347): 98-102.

47. Ducasse-Cabanot S, Cohen-Gonsaud M, Marrakchi $H$, Nguyen $M$, Zerbib D, et al. (2004) In vitro inhibition of the Mycobacterium tuberculosis $\beta$ ketoacyl-acyl carrier protein reductase MabA by isoniazid. Antimicrobl Agents Chemother 48(1): 242-249.

48. Quemard A, Lacave C, Laneelle G (1991) Isoniazid inhibition of mycolic acid synthesis by cell extracts of sensitive and resistant strains of Mycobacterium aurum. Antimicrob Agents Chemother 35(6): 10351039.

49. Wei C, Lei B, Musser J, Tu S (2003) Isoniazid Activation Defects in Recombinant Mycobacterium tuberculosis Catalase-Peroxidase (KatG) Mutants Evident in InhA Inhibitor Production. Antimicrob Agents and Chemother 47(2): 670-675.

50. Rouse D, DeVito J, Li Z, Byer H, Morris S (1996) Sitedirected mutagenesis of the katG gene of Mycobacterium tuberculosis: effects on catalase- 


\section{Epidemiology International Journal}

peroxidase activities and isoniazid resistance. Molecul Microbiol 22(3): 583-592.

51. Ramaswamy S, Musser J (1998) Molecular genetic basis of antimicrobial agent resistance in Mycobacterium tuberculosis: 1998 update. Tubercle Lung Dis 79(1): 3-29.

52. Campbell E, Korzheva N, Mustaev A, Murakami K, Nair S, et al. (2001) Structural Mechanism for Rifampicin Inhibition of Bacterial RNA Polymerase. Cell 104(6): 901-912.

53. Feklistov A, Mekler V, Jiang Q, Westblade LF, Irschik $\mathrm{H}$, et al. (2008) Rifamycins do not function by allosteric modulation of binding of Mg2+ to the RNA polymerase active center. Proc Nat Acad Sci 105(39): 14820-14825.

54. World Health Organization (2017) Drug-resistant TB.

55. Palomino J, Martin A (2014) Drug resistance mechanisms in Mycobacterium tuberculosis. Antibiotics 3(3): 317-340.

56. Zhang Y, Yew WW (2009) Mechanisms of drug resistance in Mycobacterium tuberculosis [State of the art series. Drug-resistant tuberculosis. Edited by CY. Chiang. Number 1 in the series]. Int J Tuberculosis Lung Dis 13(11): 1320-1330.

57. Velayati AA, Masjedi MR, Farnia P, Tabarsi $P$, Ghanavi J, et al. (2009) Emergence of new forms of totally drug-resistant tuberculosis bacilli: super extensively drug-resistant tuberculosis or totally drug-resistant strains in Iran. Chest 136(2): 420425.
58. Migliori G, Centis R, D'Ambrosio L, Spanevello A Borroni E, et al. (2012) Totally Drug-Resistant and Extremely Drug-Resistant Tuberculosis: The Same Disease?. Clin Infectious Dis 54(9): 1379-1380.

59. Udwadia ZF, Amale RA, Ajbani KK, Rodrigues C (2012) Totally drug-resistant tuberculosis in India. Clinical Infectious Diseases. 54(4): 579-581.

60. Catanzaro A, Rodwell TC, Catanzaro DG, Garfein RS, Jackson RL, et al. (2015) Performance comparison of three rapid tests for the diagnosis of drugresistant tuberculosis. PloS One 10(8): e0136861.

61. Rabodoarivelo MS, Imperiale B, Brandao A, Kumar P, Singh S, et al. (2015) Performance of four transport and storage systems for molecular detection of multidrug-resistant tuberculosis. PloS One 10(10): e0139382.

62. Gandhi NR, Weissman D, Moodley P, Ramathal M, Elson I, et al. (2013) Nosocomial transmission of extensively drug-resistant tuberculosis in a rural hospital in South Africa. J Infectious Dis 207(1): 917.

63. Casali N, Nikolayevskyy V, Balabanova Y, Harris SR, Ignatyeva 0 , et al. (2014) Evolution and transmission of drug-resistant tuberculosis in a Russian population. Nat genet 46(3): 279-286.

64. Kandelaki K, Lundborg CS, Marrone G (2015) Antibiotic use and resistance: a cross-sectional study exploring knowledge and attitudes among school and institution personnel in Tbilisi, Republic of Georgia. BMC Res Notes 8(1): 495. 